18,12

\title{
О магнитных состояниях зигзагообразной кромки графеновой наноленты
}

\author{
() С.Ю. Давыдов \\ Физико-технический институт им. А.Ф. Иофрфе РАН, \\ Санкт-Петербург, Россия \\ E-mail: Sergei_Davydov@mail.ru \\ Поступила в Редакцию 13 июля 2019 г. \\ В окончательной редакции 13 июля 2019 г. \\ Принята к публикации 16 июля 2019 г.
}

В рамках простой структурной модели и многоцентрового гамильтониана Андерсона получены функции Грина для атомов зигзагообразной кромки эпитаксиальной графеновой наноленты. Подробно обсуждается электронная структура свободной наноленты: найдены точные выражения для зонного спектра и плотности состояний и приведены оценки чисел заполнения и магнитных моментов. Для наноленты, сильно связанной с металлической подложкой, определены критерии возникновения магнитных моментов. Как для свободной, так и для эпитаксиальной наноленты показано, что вероятность появления магнитных моментов и их величина для атомов зигзагообразной кромки, имеющих двух ближайших соседей, выше, чем для атомов с тремя ближайшими соседями.

Ключевые слова: магнитный момент, цепочечная модель, электронная структура, плотность состояний, числа заполнения.

DOI: $10.21883 /$ FTT.2020.01.48757.502

\section{1. Введение}

В недавно вышедшей статье [1] (см. также [2]) впервые экспериментально подтверждено наличие спинового момента на зигзагообразной кромке графеновой наноленты, хотя возможность такого состояния была теоретически предсказана для кромок графита более 20 лет назад [3]. Обзор ранних публикаций по данной тематике, являющихся, во многом, продолжением предшествующих работ по фуллеренам и углеродным нанотрубкам, приведен в [4,5]. После появления пионерской публикации Новоселова, Гейма и др. [6], встал вопрос о возможности магнитного упорядочения в графеновых структурах $[7,8]$. Современное состояние проблемы обсуждается в обзоре [9] и, кратко, в Sec. IV обзора [10]. Следует отметить, что вопросу о магнитных состояниях в графене (важных, в частности, для спинтроники [11] и квантовых компьютеров [12]) уделялось гораздо меньше внимания, чем, например, электронным и фононным свойствам, что понятно, так как экспериментального доказательства магнетизма в углеродных структурах не существовало. Что же касается теории, то все перечисленные выше работы представляют собой численные расчеты. Здесь же предлагается модельный подход к проблеме, основы которого заложены в [13]. Цель настоящей работы состоит в выяснении двух вопросов: 1) при каких условиях атомы зигзагообразного края графеновой наноленты могут обладать спиновым магнитным моментом и 2) чем в этом плане отличаются атомы кромки, имеющие двух и трех ближайших соседей. Более того, здесь мы рассмотрим не только свободную, но и эпитаксиальную наноленту, так как именно последняя является элементом реальной приборной структуры.

\section{2. Цепочечная модель}

В качестве модели графеновой наноленты с зигзагообразными кромками рассмотрим структуру, изображенную на рис. 1, заимствованным из работы [13], где атомы типа $\overline{1} 1,11, \overline{1} \overline{1}, 1 \overline{1}$, имеющие двух ближай-

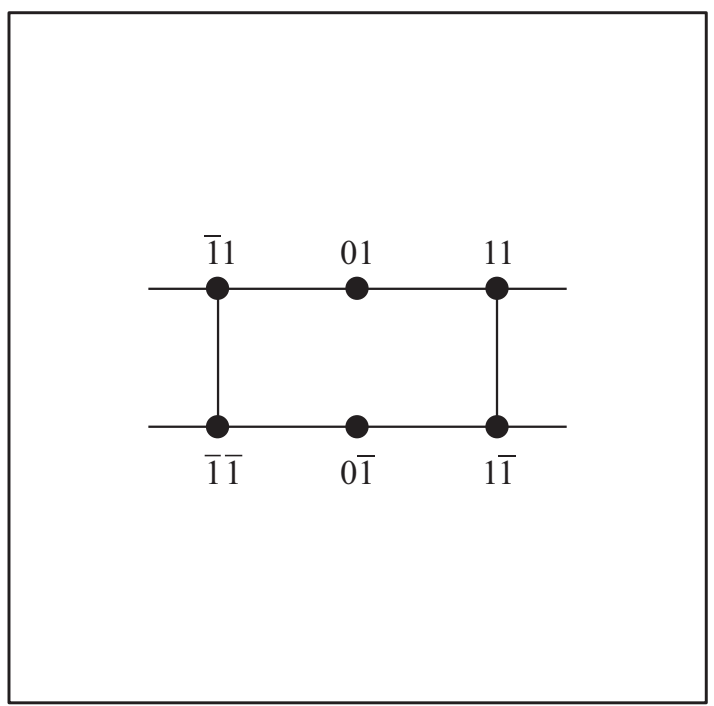

Рис. 1. Структурная модель наноленты. Атомы типа $\overline{1} 1,11$, $\overline{1} \overline{1}, 1 \overline{1}$ представляют внешние атомы зигзагообразной кромки; атомы типа 01 и $0 \overline{1}-$ внутренние атомы наноленты. 
ших соседей (б.c.), имитируют внешние атомы зигзагообразной кромки, а атомы типа 01 и $0 \overline{1}$, имеющие трех б.с., представляют внутренние атомы наноленты. Аналитическое рассмотрение начнем с самой общей постановки проблемы, считая, что нанолента находится на поверхности твердотельной подложки, так что все ее атомы являются, по сути, адатомами.

Для описания изолированного адатома углерода воспользуемся гамильтонианом Андерсона $[14,15]$, где в качестве корреляции учитывается только внутриатомное кулоновское отталкивание $U$ электронов с противоположными спинами. Гамильтониан системы имеет вид

$$
H=H_{\text {sub }}+H_{C}+H_{\text {int }},
$$

где гамильтонианы, отвечающие субстрату, атому углерода и взаимодействию между ними равны соответственно

$$
\begin{gathered}
H_{\mathrm{sub}}=\sum_{\mathbf{q}, \sigma} \varepsilon_{\mathrm{sub}}(\mathbf{q}) \hat{n}_{\mathbf{q} \sigma}, \\
H_{C}=\varepsilon \hat{n}_{\uparrow} \hat{n}_{\downarrow}, \\
H_{\mathrm{int}}=\sum_{\mathbf{q}, \sigma} V_{\mathbf{q}}\left(c_{\mathbf{q} \sigma}^{+} a_{\sigma}+\text { h.c. }\right) .
\end{gathered}
$$

Здесь $\hat{n}_{\mathbf{q} \sigma}=c_{\mathbf{q} \sigma}^{+} C_{\mathbf{q} \sigma}$ - оператор числа заполнения состояния $|\mathbf{q} \sigma\rangle$ электрона в подложке с волновым вектором $\mathbf{q}$ и проекцией спина $\sigma=(\uparrow, \downarrow), c_{\mathbf{q} \sigma}^{+}\left(c_{\mathbf{q} \sigma}\right)-$ соответствующий оператор рождения (уничтожения); $\hat{n}_{\sigma}=a_{\sigma}^{+} a_{\sigma}-$ оператор числа заполнения состояния атома углерода $\left|a_{\sigma}\right\rangle, a_{\sigma}^{+}\left(a_{\sigma}\right)$ - соответствующий оператор рождения (уничтожения); $\hat{n}=\sum_{\sigma} \hat{n}_{\sigma} ; \varepsilon_{\text {sub }}(\mathbf{q})-$ закон дисперсии электронов в подложке; $\varepsilon-$ энергия состояния $\left|p_{z}\right\rangle$ атома углерода, $V_{\mathbf{q}}$ - матричный элемент взаимодействия, спаривающий состояния $|\mathbf{q}, \sigma\rangle$ и $\left|a_{\sigma}\right\rangle$, который в дальнейшем принимается равным константе $V[14,15]$; h.c. - эрмитово сопряженные слагаемые. В рамках расширенного метода Хартри-Фока $[14,15]$ легко показать, что функция Грина, отвечающая гамильтониану Андерсона (1), имеет вид

$$
g_{\sigma}^{-1}(\omega)=\omega-\varepsilon_{\sigma}-\Lambda(\omega)+i \Gamma(\omega) .
$$

Здесь $\omega-$ энергия, $\varepsilon_{\sigma}=\varepsilon+U n_{-\sigma}$, где $n_{\sigma}=\left\langle\hat{n}_{\sigma}\right\rangle-$ число заполнения адатома углерода в состоянии $\left|a_{\sigma}\right\rangle$, скобки $\langle\ldots\rangle$ означают усреднение по основному состоянию; $\Gamma(\omega)=\pi V^{2} \rho_{\text {sub }}(\omega)-$ функция уширения квазиуровня адатома углерода, где $\rho_{\text {sub }}(\omega)$ - плотность состояний подложки; $\Lambda(\omega)=\pi^{-1} P$ $\times \int_{-\infty}^{\infty} \Gamma\left(\omega^{\prime}\right) d \omega^{\prime} /\left(\omega-\omega^{\prime}\right)-$ функция сдвига квазиуровня адатома углерода, где $P-$ символ главного значения интеграла.

Включая взаимодействия $t$ между б.с. в графене и обобщая результаты работы [13], получим соответственно для внутренних адатомов, имеющих трех б.с., и адатомов кромки, имеющих двух б.с., функции Грина вида

$$
G_{01,01 \sigma} \equiv G_{0 \sigma}(\omega, k)=g_{0 \sigma} \frac{1-g_{0 \sigma} g_{1 \sigma} t^{2} \Phi(k)}{D_{\sigma}(\omega, k)},
$$

$$
\begin{gathered}
G_{11,11 \sigma} \equiv G_{1 \sigma}(\omega, k)=g_{1 \sigma} \frac{1-g_{0 \sigma} g_{1 \sigma} t^{2}[1+\Phi(k)]}{D_{\sigma}(\omega, k)}, \\
D_{\sigma}(\omega, k)=\left[1-g_{0 \sigma} g_{1 \sigma} t^{2} \Phi(k)\right]^{2}-g_{0 \sigma} g_{1 \sigma} t^{2} .
\end{gathered}
$$

Здесь затравочные функции Грина равны $g_{0 \sigma} g_{1 \sigma}^{-1}(\omega)=$ $=\omega-\varepsilon_{0 \sigma, 1 \sigma}-\Lambda(\omega)+i \Gamma(\omega)$, где $\varepsilon_{0 \sigma, 1 \sigma}=\varepsilon+U n_{0-\sigma, 1-\sigma}$; $\Phi(k)=4 \cos ^{2}(k a)$, где $a-$ расстояние между б. с. в графене, $|k| \leq \pi / 2$ - волновой вектор. Соответствующие функциям Грина (6) и (7) плотности состояний есть $\rho_{j \sigma}(\omega)=-\pi^{-1} \operatorname{Im} G_{j \sigma}(\omega)$, где $G_{j \sigma}=N^{-1} \sum_{k} G_{j \sigma}(\omega, k)$, $j=0,1$ и $N-$ число $k$-состояний в зоне Бриллюэна, а числа заполнения при нулевой температуре равны $n_{j \sigma}=\int_{-\infty}^{E_{F}} \rho_{j \sigma}(\omega) d \omega$, где $E_{F}-$ уровень Ферми. Введем теперь магнитные моменты адатомов $m_{j}=n_{j \uparrow}-n_{j \downarrow}$. Основная задача настоящей работы состоит в определении условий, при которых $m_{j} \neq 0$ и ответа на вопрос, есть ли и чем обусловлены различия между моментами $m_{0}$ и $m_{1}$.

\section{3. Свободная нанолента}

\section{1. Энергетические зоны и плотности состояний}

Рассмотрим свободную (не связанную с подложкой) наноленту, которой отвечает функция Грина $g_{j \sigma}^{-1}(\omega)=\Omega_{j \sigma}+i 0^{+}$, где $\Omega_{j \sigma}=\omega-\varepsilon_{j \sigma}$. Положим $\varepsilon_{0 \sigma}+\varepsilon_{1 \sigma}=2 \varepsilon_{\sigma}, \varepsilon_{0 \sigma}-\varepsilon_{1 \sigma}=2 \Delta_{\sigma}$, так что $\varepsilon_{0 \sigma, 1 \sigma}=\varepsilon_{\sigma} \pm \Delta_{\sigma}$.

Электронный спектр свободной наноленты определяется корнями уравнения $D_{\sigma}(\omega, k)=0$, равными

$$
\begin{aligned}
\Xi_{\sigma p}(k) & \equiv \sqrt{\left(\omega_{\sigma p}(k)-\varepsilon_{\sigma}\right)^{2}-\Delta_{\sigma}^{2}} \\
& = \pm t[\sqrt{1+4 \Phi(k)} \pm 1] / 2
\end{aligned}
$$

где $\left(\omega-\varepsilon_{\sigma}\right)^{2}>\Delta_{\sigma}^{2}$. Имеются, таким образом, четыpe зоны $\Xi_{\sigma 1}(k)=-\Xi_{\sigma 4}(k), \Xi_{\sigma 2}(k)=-\Xi_{\sigma 3}(k)$ для каждой проекции спина. Переходя к реальному спектру $\omega_{\sigma p}(k)$, отметим, что на границе зоны Бриллюэна имеем $\omega_{\sigma 1,4}(\pi / 2 a)=\varepsilon_{\sigma} \pm \sqrt{\Delta_{\sigma}^{2}+t^{2}}$ и $\omega_{\sigma 2,3}(\pi / 2 a)=\varepsilon_{\sigma} \pm\left|\Delta_{\sigma}\right|$, в центре зоны $\omega_{\sigma 1,4}(0)=\varepsilon_{\sigma} \pm \sqrt{\Delta_{\sigma}^{2}+t^{2}(\sqrt{17}+1)^{2} / 4}$ и $\omega_{\sigma 2,3}(0)=\varepsilon_{\sigma} \pm \sqrt{\Delta_{\sigma}^{2}+t^{2}(\sqrt{17}-1)^{2} / 4}$. Электронный спектр для немагнитного случая $\left(\Delta_{\sigma}=0\right)$ представлен на рис. 2, где нулю энергии соответствует точка Дирака.

Обобщая результаты [13], перепишем функции Грина (6) и (7) в виде

$$
\begin{gathered}
G_{0 \sigma}\left(\Omega_{\sigma}\right)=\frac{\Omega_{1 \sigma}}{2}\left(I_{\sigma-}+I_{\sigma+}\right), \\
G_{1 \sigma}\left(\Omega_{\sigma}\right)=\frac{\Omega_{0 \sigma}}{\Omega_{1 \sigma}} G_{0 \sigma}\left(\Omega_{\sigma}, k\right)+\frac{\Omega_{0 \omega} t}{2 \xi_{\sigma}}\left(I_{\sigma+}-I_{\sigma-}\right),
\end{gathered}
$$

где

$$
I_{\sigma \pm}\left(\Omega_{\sigma}\right)= \begin{cases}\frac{1}{\sqrt{d_{\sigma \pm}^{2}-4 t^{4}}}, & d_{\sigma \pm}^{2}>4 t^{4} \\ \frac{-i}{\sqrt{4 t^{4}-d_{\sigma \pm}^{2}}}, & d_{\sigma \pm}^{2}<4 t^{4}\end{cases}
$$




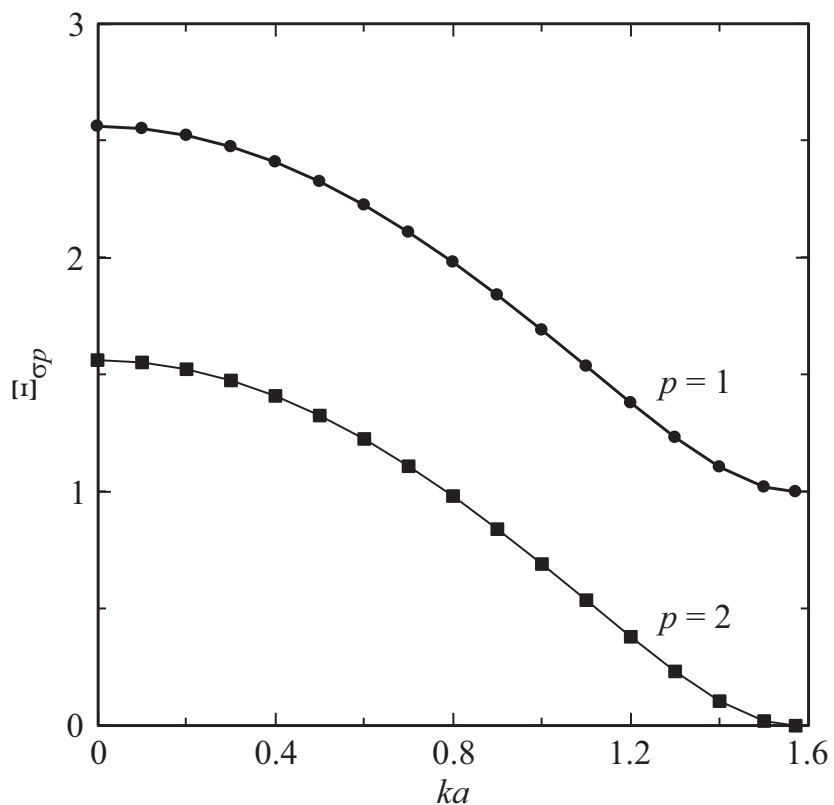

Рис. 2. Свободная нанолента: энергетические зоны $\Xi_{\sigma p}(k)$ для немагнитного случая. Нуль энергии соответствует точке Дирака, энергия измеряется в ед. $t$. Приведены только зоны, отвечающие положительной энергии.

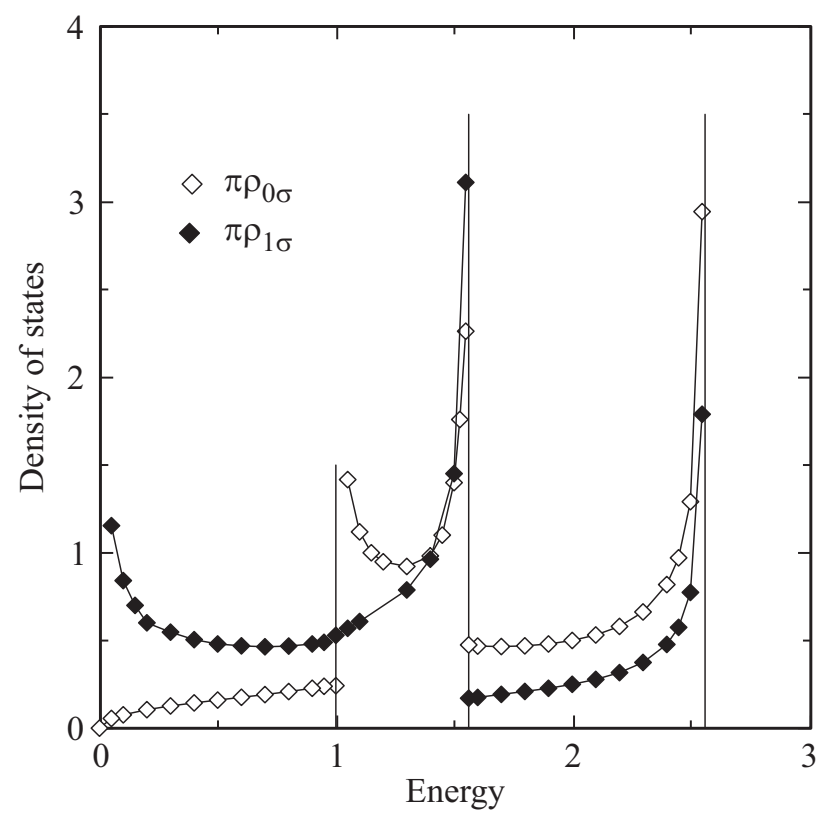

Рис. 3. Свободная нанолента: плотности состояний $\rho_{0 \sigma}$ (светлые ромбы) и $\rho_{1 \sigma}$ (темные ромбы) для одной проекции спина в немагнитном случае. Нуль энергии соответствует точке Дирака, энергия измеряется в ед. $t$, плотности состояний в ед. $t^{-1}$. Приведены только правые половины симметричных плотностей состояний, отвечающие положительной энергии.

$d_{\sigma \pm}=\xi_{\sigma}^{2}-\Delta_{\sigma}^{2} \pm t \xi_{\sigma}-2 t^{2}, \varepsilon_{\sigma}=\sqrt{\Omega_{\sigma}^{2}-\Delta_{\sigma}^{2}}, \Omega_{\sigma}=\omega-\varepsilon_{\sigma}$. Так как $\rho_{j \sigma}\left(\Omega_{\sigma}\right)=-\pi^{-1} \operatorname{Im} G_{j \sigma}\left(\Omega_{\sigma}\right)$, из выражения (12) следует, что отличные от нуля плотности состояний существуют только при $d_{\sigma \pm}^{2}<4 t^{4}$. Проделав соответ- ствующие алгебраические преобразования, получим для $\rho_{j \sigma}\left(\Omega_{\sigma}\right)$ следующие выражения:

$$
\begin{gathered}
\rho_{0 \sigma}\left(\Omega_{\sigma}\right)=\frac{\Omega_{1 \sigma} A^{+}\left(\Omega_{\sigma}\right)}{2 \pi} \\
\rho_{1 \sigma}\left(\Omega_{\sigma}\right)=\frac{\Omega_{0 \sigma}}{\Omega_{1 \sigma}} \rho_{0 \sigma}\left(\Omega_{\sigma}\right)+\frac{t \Omega_{0 \sigma} A^{-}\left(\Omega_{\sigma}\right)}{2 \pi \xi_{\sigma}} \\
A^{ \pm}\left(\Omega_{\sigma}\right)=\frac{\Theta\left(4 t^{4}-d_{\sigma+}^{2}\right)}{R_{\sigma+}} \pm \frac{\Theta\left(4 t^{4}-d_{\sigma-}^{2}\right)}{R_{\sigma-}},
\end{gathered}
$$

где $R_{\sigma \pm}=\sqrt{4 t^{4}-d_{\sigma \pm}^{2}}, \Omega_{0 \sigma, 1 \sigma}=\Omega_{\sigma} \mp \Delta_{\sigma}$. Плотности состояний для одной проекции спина в немагнитном случае $\left(\Delta_{\sigma}=0\right)$ представлены на рис. 3 для положительных энергий, нуль энергии совмещен с точкой Дирака. Обратим внимание, что в области точки Дирака для атомов типа 1 плотность состояний $\rho_{1 \sigma} \rightarrow \infty$, а для атомов типа 0 имеем $\rho_{0 \sigma} \rightarrow 0$.

\section{2. Числа заполнения и спиновые моменты}

Теперь следует вычислить числа заполнения $n_{j \sigma}$. При этом получим систему четырех самосогласованных уравнений вида $n_{0 \sigma}=Q_{0}\left(n_{0 \sigma}, n_{1 \sigma}\right)$ и $n_{1 \sigma}=Q_{1}\left(n_{0 \sigma}, n_{1 \sigma}\right)$, где $Q_{0,1}(\ldots)$ - некоторые функции. Это весьма сложная задача: нужно не только определить область параметров, для которых возможны магнитные решения вида $n_{j \sigma} \neq n_{j-\sigma}$ (необходимое условие), но и выявить интервал внутри области, где такое решение энергетически выгодно (достаточное условие). Подчеркнем: в общем виде такая задача была решена лишь для случая одной магнитной примеси в немагнитной матрице [14], тогда как для двух взаимодействующих примесей получить такое решение уже не удалось [16]. Поэтому здесь мы не будем рассматривать общую задачу, а прибегнем к ряду упрощений.

Сразу же учтем, что в силу сохранения электронов в наноленте $n_{0}+n_{1}=2$. Более того, будем считать, что наличие магнитных состояний не меняет чисел заполнения атомов наноленты $n_{0}$ и $n_{1}$, так что, как и в бесконечном графеновом листе, имеем $n_{0}=n_{1}=1$. Легко показать, что эти равенства реализуется при условии $\varepsilon_{D}=\varepsilon+U / 2=0$ (точка Дирака) и $E_{F}=\varepsilon_{D}$ (недопированная нанолента). Отсюда вытекают тождества $\sum_{\sigma} \varepsilon_{\sigma}=0$ и $\sum_{\sigma} \Delta_{\sigma}=0$. Положим $\varepsilon_{\uparrow}=-\varepsilon_{\downarrow}=e \geq 0$, $\Delta_{\uparrow}=-\Delta_{\downarrow}=\Delta>0$.

Начнем с простейшего случая, когда $\varepsilon_{\uparrow}=\varepsilon_{\downarrow}=0$. При этом должны выполняться равенства $n_{0 \downarrow}+n_{1 \downarrow}=1$ и $n_{0 \uparrow}+n_{1 \uparrow}=1$, а нетривиальное решение $\Delta \neq 0$ возможно только при условии $n_{0 \downarrow}=n_{1 \uparrow} \quad$ и $n_{0 \uparrow}=n_{1 \downarrow}$, соответствующем антиферромагнитному упорядочению. Вводя $n_{0 \uparrow, 0 \downarrow}=0.5 \mp v_{0}, \quad n_{1 \uparrow, 1 \downarrow}=0.5 \mp v_{1}$, получим $v_{0}=-v_{1}=v \quad$ и $\Delta_{\uparrow, \downarrow}= \pm 2 U \nu$. В случае ферромагнитного упорядочения, когда $n_{0 \downarrow}=n_{1 \downarrow}$ и $n_{0 \uparrow}=n_{1 \uparrow}$, имеем $\quad v_{0}=v_{1}=v$, так что $\Delta=0$ и $\quad \varepsilon_{\uparrow, \downarrow}=\mp 2 U v$. В обоих рассмотренных выше случаях $\left|m_{0}\right|=\left|m_{1}\right|$. Для нашей задачи больший интерес представляет ситуация, когда $\left|m_{0}\right| \neq\left|m_{1}\right|$. Пусть, например, $m_{0}=0$, 
$m_{1} \neq 0$, т.е. магнитным моментом обладает только атом кромки. Такому условию отвечают $\Delta_{\uparrow, \downarrow}=\mp U v_{1}$, $\varepsilon_{\uparrow, \downarrow}= \pm U v_{1}$. Здесь мы рассмотрим случай, когда $n_{0 \uparrow, 0 \downarrow}=0.5 \mp v_{0}, \quad n_{1 \uparrow, 1 \downarrow}=0.5 \mp v_{1}$, т. е. $\quad \varepsilon_{\uparrow}=\varepsilon_{\downarrow}=0$ и $\Delta_{\uparrow, \downarrow}=\mp U\left(v_{1}-v_{0}\right)$, что соответствует ферримагнитному упорядочению. Пренебрегая величинами $\sim(\Delta / t)^{2}$ и положив $\rho_{0,1 \sigma}(\omega)=\rho_{0,1}^{\prime}(\omega)+\Delta_{\sigma} r_{0,1 \sigma}(\omega)$, из (13) и (14) получим следующие выражения:

$$
\begin{gathered}
\rho_{0 \sigma}^{\prime}(\omega)=\frac{\omega A^{+}(\omega)}{2 \pi}, \rho_{1 \sigma}^{\prime}(\omega)=\rho_{0 \sigma}^{\prime}(\omega)+\frac{t A^{-}(\omega)}{2 \pi}, \\
r_{0 \sigma}(\omega)=\frac{A^{+}(\omega)}{2 \pi}, r_{1 \sigma}(\omega)=-r_{0 \sigma}-\frac{t A^{-}(\omega)}{2 \pi \omega} \\
A^{ \pm}(\omega)=\frac{\Theta\left(4 t^{4}-d_{+}^{2}\right)}{\bar{R}_{+}} \pm \frac{\Theta\left(4 t^{4}-d_{-}^{2}\right)}{\bar{R}_{-}}
\end{gathered}
$$

где $\quad \bar{R}_{ \pm}=\sqrt{4 t^{4}-d_{ \pm}^{2}}, \quad d_{ \pm}=\omega^{2} \pm t \omega-2 t^{2}, \quad$ индекс $\sigma$ в (15) означает, что плотности состояний относятся к одному спиновому состоянию.

Определим теперь значения чисел заполнения $n_{j \sigma}=n_{j \sigma}^{\prime}+\Delta_{\sigma} v_{j \sigma}, \quad$ где $n_{j \sigma}^{\prime}=\int_{-\infty}^{0} \rho_{j \sigma}^{\prime}(\omega) d \omega \quad$ и $v_{j \sigma}=$ $=\int_{-\infty}^{0} r_{j \sigma}(\omega) d \omega$. Легко показать, что $n_{0 \sigma}^{\prime}=\left(J_{-}+J_{+}\right) / 2 \pi$, $n_{1 \sigma}^{\prime}=n_{0 \sigma}^{\prime}+t\left(S_{+}-S_{-}\right) / 2 \pi, v_{0 \sigma}=\left(S_{-}+S_{+}\right) / 2 \pi, v_{1 \sigma}=$ $=-v_{0 \sigma}-t\left(P_{-}+P_{+}\right) / 2 \pi$, где значения интегралов $J_{ \pm}$, $S_{ \pm}, P_{ \pm}$приведены в Приложении. Оставляя только главные члены $\sim \sqrt{\Delta / t}$, находим окончательно

$$
\begin{aligned}
& n_{0 \uparrow, 0 \downarrow} \approx \frac{1}{2}\left(1 \mp \frac{\eta \sqrt{\Delta / t}}{\pi(1-\eta) \sqrt{1-\eta^{2}}}\right), \\
& n_{1 \uparrow, 1 \downarrow}^{\prime}=\frac{1}{2}\left[1 \mp \frac{9 \eta \sqrt{\Delta / t}}{8 \pi(1-\eta) \sqrt{1-\eta^{2}}}\right] .
\end{aligned}
$$

Отсюда получаем (см. Приложение) $\left|m_{1}\right|>\left|m_{0}\right|$. Этого, вообще говоря, и следовало ожидать, так как величина наведенного спинового момента пропорциональна $U$ и плотности состояний системы на уровне Ферми $[17,18]$. В области $E_{F}=\varepsilon_{D}$ имеем соотношение $\rho_{1 \sigma}>\rho_{0 \sigma}$ (что легко усмотреть из рис. 3 ), откуда и получаем $\left|m_{1}\right|>\left|m_{0}\right|$.

\section{4. Эпитаксиальная нанолента}

Рассмотрим теперь графеновую наноленту, сформированную на металле. Полагая плотность состояний подложки $\rho_{\text {sub }}(\omega)=$ const, получим $\Lambda(\omega)=0$ и $\Gamma(\omega)=\Gamma_{m}=$ const [14-16]. Будем считать, что имеет место режим сильной связи наноленты с подложкой, когда $\Gamma_{m} \gg t$. Тогда, разложив функции Грина (6) и (7) с точностью до $t^{2} / \Gamma_{m}^{2}$ и проинтегрировав их по $k$, получим

$$
\begin{gathered}
G_{0 \sigma}(\omega) \approx g_{0 \sigma}\left(1+3 g_{0 \sigma} g_{1 \sigma} t^{2}\right), \\
G_{1 \sigma}(\omega) \approx g_{1 \sigma}\left(1+2 g_{0 \sigma} g_{1 \sigma} t^{2}\right),
\end{gathered}
$$

где $g_{j \sigma}^{-1}=\Omega_{j \sigma}+i \Gamma_{m}, \Omega_{j \sigma}=\omega-\varepsilon_{j \sigma}$. Отметим, что численные множители перед вторыми слагаемыми в скобках равны числу б. с. $z_{j}$ атомов $0\left(z_{0}=3\right)$ и $1\left(z_{1}=2\right)$.

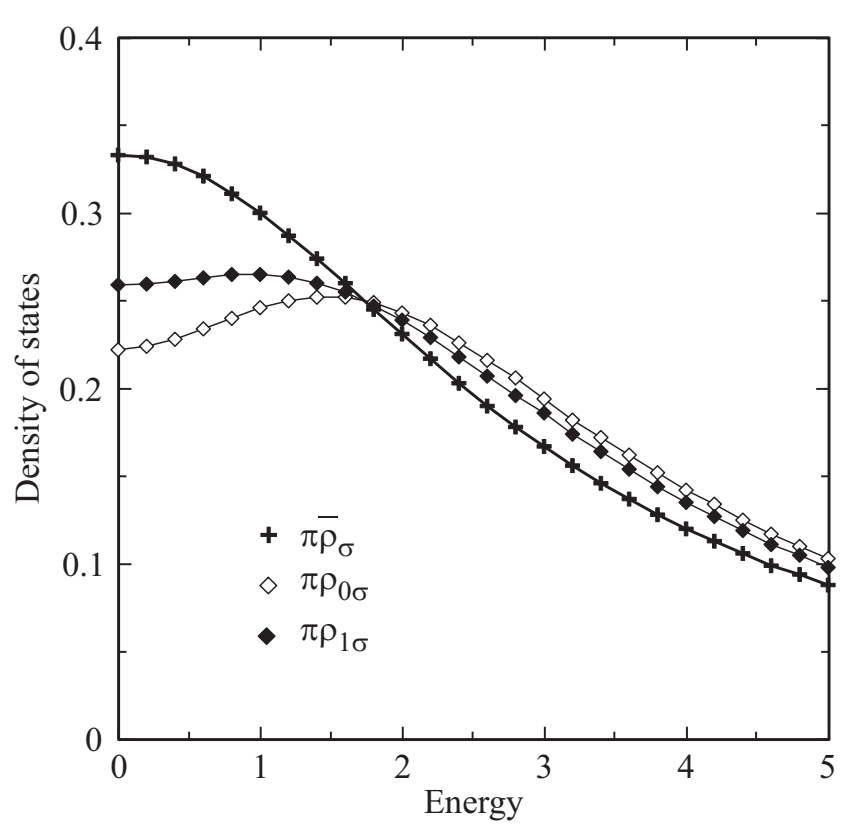

Рис. 4. Нанолента на металлической подложке: плотности состояний $\bar{\rho}_{\sigma}$ (крестики), $\rho_{0 \sigma}$ (светлые ромбы) и $\rho_{1 \sigma}$ (темные ромбы) для одной проекции спина в немагнитном случае в режиме сильной связи с подложкой $\Gamma_{m}=3 t$. Нуль энергии соответствует точке Дирака, энергия измеряется в ед. $t$, плотности состояний - в ед. $t^{-1}$. Приведены только правые половины симметричных плотностей состояний, отвечающие положительной энергии.

Функциям Грина (19) соответствуют плотности состояний вида

$$
\rho_{j \sigma}(\omega) \approx \bar{\rho}_{j \sigma}(\omega)\left(1+z_{j} t^{2} \frac{2 \Omega_{0 \sigma} \Omega_{1 \sigma}+\Omega_{0 \sigma}^{2}-\Gamma_{m}^{2}}{\left(\Omega_{0 \sigma}^{2}+\Gamma_{m}^{2}\right)\left(\Omega_{1 \sigma}^{2}+\Gamma_{m}^{2}\right)}\right),
$$

где

$$
\bar{\rho}_{j \sigma}(\omega)=\frac{1}{\pi} \frac{\Gamma_{m}}{\Omega_{j \sigma}^{2}+\Gamma_{)}^{2} m} .
$$

является плотностью состояний изолированного $j$-го адатома углерода. На рис. 4 представлены функции $\rho_{0 \sigma}(\omega)$ и $\rho_{1 \sigma}(\omega)$ в немагнитном случае $\varepsilon_{0 \sigma}=\varepsilon_{1 \sigma}$ $=\varepsilon_{0-\sigma}=\varepsilon_{1-\sigma}=e^{*}$, там же изображена плотность состояний изолированного адатома $\bar{\rho}_{\sigma}(\omega)$. Из рис. 4 следует, что вместо одногорбой кривой $\bar{\rho}_{j \sigma}(\omega)$ нанолента в режиме сильной связи характеризуется двугорбыми кривыми с минимумами $\left(\rho_{j \sigma}\right)_{\min }=\left(\pi \Gamma_{m}\right)^{-1}\left(1-z_{j} t^{2} / \Gamma_{m}^{2}\right)$ при $\omega=e^{*}$. Сравнение со свободной нанолентой показывает (рис. 3), во-первых, что под действием металлической подложки все расходимости плотностей состояний исчезают. Во-вторых, резкие различия между функциями $\rho_{0 \sigma}(\omega)$ и $\rho_{1 \sigma}(\omega)$ нивелируются. И, наконец, понижение плотности состояний $\rho_{1 \sigma}(\omega)$ вблизи точки Дирака уменьшает вероятность возникновения намагниченности.

Переходя к числам заполнения, рассмотрим для начала задачу о спиновом моменте изолированного адатома как предельном случае режима сильной связи. 
Согласно (21), число заполнения такого адатома равно $\bar{n}_{\sigma}=\pi^{-1} \operatorname{arcctg}\left[\left(\varepsilon_{\sigma}-E_{F}\right) / \Gamma_{m}\right]$ (здесь индекс $j$ опускаем). Вновь принимая $2 \varepsilon+U=0$, можем записать $\varepsilon_{\sigma}=U v_{-\sigma}$, где $v_{\sigma}=n_{\sigma}=0.5$. Тогда для спинового момента $\bar{m}=\bar{n}_{\uparrow}-\bar{n}_{\downarrow}$ получим уравнение

$$
\begin{aligned}
\bar{m} & =v_{\uparrow}-v_{\downarrow} \\
& =\frac{1}{\pi}\left(\operatorname{arcctg} \frac{U v_{\downarrow}-E_{F}}{\Gamma_{m}}-\operatorname{arcctg} \frac{U v_{\uparrow}-E_{F}}{\Gamma_{m}}\right) .
\end{aligned}
$$

Полагая для простоты $E_{F}=0$, получаем $\bar{n}=$ $=\bar{n}_{\uparrow}+\bar{n}_{\downarrow}=1, v_{\uparrow}=-v_{\downarrow}=v$ и приходим к уравнению $\operatorname{tg} \pi v=\left(U / \Gamma_{m}\right) v$, которое имеет нетривиальное решение $v \neq 0$ при

$$
U / \pi \Gamma_{m}>1 .
$$

Отметим, что для одиночного адатома неравенство (23) является точным [14].

Переходя от изолированного адатома к наноленте, перепишем для начала функции Грина (19) в кластерном виде: $G_{0 \sigma}(\omega) \approx g_{0 \sigma}\left(1-3 g_{0 \sigma} g_{1 \sigma} t^{2}\right)^{-1}$ и $G_{1 \sigma}(\omega) \approx g_{1 \sigma}\left(1-2 g_{0 \sigma} g_{1 \sigma} t^{2}\right)^{-1}$. Эти функции Грина можно приписать адсорбированным димерам с энергиями перехода между б.с., равными соответственно $t \sqrt{3}$ и $t \sqrt{2}$. Задача о магнитных состояниях адсорбированного на металле димера рассматривалась нами еще в работе [19] (см. также [20]). Воспользовавшись полученными там результатами, условие возникновения конечного спинового момента на адатоме типа $j$ можно представить в виде

$$
U / \pi \Gamma_{m}>B_{j}, \quad B_{j}=\left(t \sqrt{z_{j}} / \Gamma_{m}\right)\left[\operatorname{arcctg}\left(t \sqrt{z_{j}} / \Gamma_{m}\right)\right]^{-1} .
$$

Так как $B_{j}>1$, условие появления спинового момента для адатомов наноленты (24) жестче, чем условие для изолированного адатома углерода (23). С ростом $z_{j}$ значение $B_{j}$ увеличивается, так что вероятность появления спинового момента у адатомов типа 1 выше, чем у адатомов типа 0. Подчеркнем, что неравенства (23) и (24) являются необходимыми условиями существования спинового момента. Интересно также отметить, что условие (24) для адсорбированного димера $\left(z_{j}=1\right)$ получено в [19] без какого-либо ограничения, налагаемого на параметр $\Gamma_{m}$. Тогда в пределе $\Gamma_{m} \rightarrow 0$ получим неравенство

$$
U>t \sqrt{z_{j}}
$$

которое можно трактовать, как приближенное условие возникновения спинового момента в свободной наноленте.

В случае наноленты, сформированной на полупроводниковой подложке, возможны различные варианты, в той или иной степени близкие и свободной наноленте, и наноленте на металле. Действительно, в модели ХалдейнаАндерсона [21] имеем $\rho_{\text {sub }}(\omega)=$ const, $\Gamma_{m}=$ const при $|\omega| \geq E_{g} / 2$ и $\rho_{\text {sub }}(\omega)=0, \Gamma_{m}=0$ при $|\omega|<E_{g} / 2$, где $E_{g}$ - ширина запрещенной зоны, центр которой совпадает с началом отсчета энергии. Тогда, если точка
Дирака перекрывается с разрешенными зонами и отстоит далеко от краев этих зон, имеем металлическую ситуацию. Если же точка Дирака попадает в область запрещенной зоны, то ситуация близка к свободному состоянию наноленты (см., например, [20,22,23]). Поэтому здесь случай полупроводниковой подложки специально не рассматривается.

\section{5. Заключение}

Приведем некоторые энергетические оценки. Выигрыш в энергии системы при переходе от состояния немагнитного к магнитному состоянию связан с кулоновским вкладом $E_{C}=U n_{\uparrow} n_{\downarrow}$. При $n_{\uparrow}+n_{\downarrow}=n$ в немагнитном случае $\left(n_{\uparrow}=n_{\downarrow}=n / 2\right.$ имеем $E_{C}=U n^{2} / 4$, в магнитном случае $\left(n_{\uparrow, \downarrow}=n / 2 \pm v\right)$ получаем $E_{C}=$ $=U n^{2} / 4-U v^{2}$. Отметим, что для графена согласно [24] отношение $t / U \approx 0.30-0.16$ при $t=2.8 \mathrm{eV}$, так что кулоновское отталкивание $U$ является главным параметром в задаче о свободной наноленте.

В случае эпитаксиальной наноленты появляется дополнительный параметр $\Gamma_{m}$ (металлическая подложка), оценить который не так просто. Дело в том, что величина $\Gamma_{m}$ зависит от способа формирования или нанесения наноленты на подложку. В случае квазисвободной наноленты $\Gamma_{m}$ определяется взаимодействием Ван-дерВаальса $(\sim 0.1 \mathrm{eV})$; если же нанолента сильно связана с подложкой, то в игру вступает ковалентное взаимодействие $(\sim 10 \mathrm{eV}$ для $d$-металлов) (см. соответствующие оценки в [25]).

Подводя итоги, отметим, что любое взаимодействие атомов углерода (как между собой, так и с подложкой) понижает вероятность появления спинового момента (см. неравенства (23)-(25)). Если же спиновый момент возникает, то чем сильнее такое взаимодействие, тем меньше его величина. Так как результирующий эффект межатомного взаимодействия пропорционален числу б.с., вероятность появления магнитных моментов и их величина для атомов зигзагообразной кромки с двумя б. с. выше, чем для атомов с тремя б.с.

\section{Приложение}

Для нахождения чисел заполнения необходимо вычислить интегралы:

$$
\begin{gathered}
J_{-}=\int_{\omega_{1}(\pi / 2 a)}^{\omega_{1}(0)} \frac{\omega d \omega}{\bar{R}_{-}(\omega)}, \quad S_{-}=\int_{\omega_{1}(\pi / 2 a)}^{\omega_{1}(0)} \frac{d \omega}{\bar{R}-(\omega)}, \\
P_{-}=\int_{\omega_{1}(\pi / 2 a)}^{\omega_{1}(0)} \frac{d \omega}{\omega \bar{R}_{-}(\omega)} ; \\
J_{+}=\int_{\omega_{2}(\pi / 2)}^{\omega_{2}(0)} \frac{\omega d \omega}{\bar{R}_{+}(\omega)}, \quad S_{+}=\int_{\omega_{2}(\pi / 2)}^{\omega_{2}(0)} \frac{d \omega}{\bar{R}_{+}(\omega)},
\end{gathered}
$$

Физика твердого тела, 2020, том 62, вып. 1 


$$
P_{+}=\int_{\omega_{2}(\pi / 2)}^{\omega_{2}(0)} \frac{\omega d \omega}{\omega \bar{R}_{+}(\omega)},
$$

где $\quad \omega_{1}(0)=t(\sqrt{17}+1) / 2, \quad \omega_{2}(0)=t(\sqrt{17}-1) / 2$, $\omega_{1}(\pi / 2 a)=t, \omega_{2}(\pi / 2 a)=\Delta$. Используя справочник [26] (формулы 3.147-3.149), получим для интегралов $J_{-}$, $S_{-}$и $P_{-}$следующие выражения:

$$
\begin{gathered}
J_{-}=2[(1+\eta) \Pi(-\eta, \eta)-\eta K(\eta)], \\
S_{-}=\left(2 / \omega_{1}(0)\right) K(\eta), \\
P_{-}=\left(1 / 2 t^{2}\right)[(1+\eta) \Pi(1, \eta)-K(\eta)], \\
J_{+}=2[(1+\eta) \Pi(\varphi,-\eta, \eta)-F(\varphi, \eta)], \\
S_{+}=\left(2 / \omega_{1}(0)\right) F(\varphi, \eta), \\
P_{+}=\left(1 / 2 t^{2}\right)[(1+\eta) \Pi(\varphi, 1, \eta)-\eta F(\varphi, \eta)],
\end{gathered}
$$

где $K(\eta)$ и П $(n, \eta)$ - полные эллиптические интегралы 1-го и 3-го рода, $F(\varphi, \eta)$ и П $(\varphi, n, \eta)$ - неполные эллиптические интегралы 1-го и 3 -го рода $[26,27]$,

$$
\eta=(\sqrt{17}-1) /(\sqrt{17}+1)
$$

И

$$
\varphi=\arcsin \left(\sqrt{\left[1-\Delta / \omega_{2}(0)\right] /\left[1+\Delta / \omega_{1}(0)\right]}\right) .
$$

Для дальнейшего нужно разложить интегралы $J_{+}, S_{+}$ и $P_{+}$по $\varphi=\pi / 2-\beta$, где $\beta \approx \sqrt{\Delta / t} / 2 \ll 1$. Исходя из определения интегралов $F(\varphi, \eta)$, П $(\varphi, n, \eta)$ и полагая

$$
\begin{gathered}
F(\varphi, \eta)=K(\eta)-\kappa(\beta, \eta), \\
\kappa(\beta, \eta)=\int_{1-\beta^{2} / 2}^{1} \frac{d x}{\sqrt{\left(1-x^{2}\right)\left(1-\eta^{2} x^{2}\right)}}, \\
\Pi(\varphi, n, \eta)=\Pi(n, \eta)-\pi(\beta, n, \eta), \\
\pi(\beta, n, \eta)=\int_{1-\beta^{2} / 2}^{1} \frac{d x}{\left(1+n x^{2}\right) \sqrt{\left(1-x^{2}\right)\left(1-\eta^{2} x^{2}\right)}}
\end{gathered}
$$

получим

$$
\begin{gathered}
\kappa(\beta, \eta) \approx \sqrt{\Delta / t} / 4 \sqrt{1-\eta^{2}}, \\
\pi(\beta, n, \eta) \approx \sqrt{\Delta / t} / 4(1+n) \sqrt{1-\eta^{2}} .
\end{gathered}
$$

Таким образом, сохраняя только члены $\sim \sqrt{\Delta / t}$ и учитывая соотношение $\Pi(-\eta, \eta)=[\pi+2(1+\eta) \times$ $K(\eta)] / 4(1+\eta)[27]$, приходим к формулам (18).

\section{Конфликт интересов}

Автор заявляет, что у него нет конфликта интересов.

\section{Список литературы}

[1] M.K. Slota, A. Keerthi, W.K. Myers, E. Tretyakov, M. Baumgarten, A. Ardavan, H. Sadeghi, C.J. Lambert, A. Narita, K. Müllen, L. Bogani. Nature 557, 691 (2018).

[2] F. Luis, E. Coronado. Nature 557, 645 (2018).

[3] M. Fujita, K. Wakabayashi, K. Nakada, K. Kusakabe. J. Phys. Soc. Jpn 65, 1920 (1996).

[4] Т.Л. Макарова. ФТП 38, 641 (2004).

[5] E. Kan, Z. Li, J. Yang. Nano 3, 433 (2006).

[6] K.S. Novoselov, A.K. Geim, S.V. Morozov, D. Jiang, Y. Zhang, S.V. Dubonos, I.V. Grigorieva, A.A. Firsov. Science 306, 666 (2004).

[7] Y.-W. Son, M.L. Cohen, S.G. Louie. Nature 444, 347 (2006).

[8] V. Barone, O. Hod, G.E. Scuseria. Nano Lett. 6, 2748 (2006).

[9] O.V. Yazyev. Rep. Prog. Phys. 73, 056501 (2010).

[10] V. Meunier, A.G. Souza Filho, E.B. Barros, M.S. Dresselhaus. Rev. Mod. Phys. 88, 025005 (2016).

[11] D. Pesin, A.H. MacDonald. Nature Mater. 11, 409 (2012).

[12] P. Recher, B. Trauzettel. Nanotechnology 21, 302001 (2010).

[13] С.Ю. Давыдов. ФТП (2019). В печати.

[14] P.W. Anderson. Phys. Rev. 124, 41 (1961).

[15] Ч. Киттель. Квантовая теория твердых тел. Наука, М. (1967). Гл. 18. 492 с.

[16] S. Alexander, P.W. Anderson. Phys. Rev. 133, A1594 (1964).

[17] Дж. Займан. Принципы теории твердого тела. Мир, М. (1974). 472 c.

[18] Т. Мория. Спиновые флуктуации в магнетиках с коллективизированными электронами. Мир, М. (1988). 288 с.

[19] С.Ю. Давыдов. ФТТ 21, 2283 (1979).

[20] С.Ю. Давыдов. Теория адсорбции: метод модельных гамильтонианов. Изд-во СПбГЭТУ „ЛЭТИ“, СПб. (2013). 235 c. twirpx.com/file/1596114/

[21] F.D.M. Haldane, P.W. Anderson. Phys. Rev. B 13, 2553 (1976).

[22] С.Ю. Давыдов. ФТТ 54, 2193 (2012).

[23] С.Ю. Давыдов. ФТТ 58, 1182 (2016).

[24] T.O. Wehling, E. Sasioglu, C. Friedrich, A.I. Lichtenstein, M.I. Katsnelson, S. Blügel. Phys. Rev. Lett. 106, 236805 (2011).

[25] С.Ю. Давыдов. ФТТ 60, 808 (2018).

[26] И.С. Градштейн, И.М. Рыжик. Таблицы интегралов, сумм, рядов и произведений. Наука, М. (1971). 1108 с.

[27] P.F. Bird, M.D. Friedman. Handbook of Elliptic Integrals for Engineers and Scientists. Springer, Berlin-Heidelberg-N.Y. (1971). $358 \mathrm{p}$.

Редактор К.В. Емцев 